\title{
Vers une simplification du système fiscal?
}

\author{
Éric Bélanger $^{\mathrm{a}}$, Marie-Eve Tremblay ${ }^{\mathrm{b}}$
}

RÉSUMÉ. Il est généralement admis que les lois fiscales entrent dans la catégorie des lois les plus complexes à lire. Les auteurs illustreront cette complexité avec des exemples simples que les non-initiés peuvent aisément comprendre. Ils salueront quelques initiatives récentes de simplification et entameront une réflexion sur les difficultés de simplifier le système actuel, bien que cela soit souhaitable.

ABSTRACT. It is generally accepted that tax laws fall into the category of the most complex laws to read. The authors will illustrate this complexity with simple examples that the uninitiated can easily understand. They will welcome some recent initiatives for simplification and start to think on the difficulties of simplifying the current system, although this is desirable.

\section{Introduction}

C’est en 1917 que le ministre des Finances de l'époque, monsieur Thomas White, annonça la "Loi de l'impôt de guerre sur le revenu » qui permettait au gouvernement fédéral de prélever un impôt direct sur le revenu des sociétés et des particuliers afin de payer les dépenses extraordinaires occasionnées par la Première Guerre mondiale. La version initiale était très simple puisqu'elle ne contenait qu'une dizaine de pages. Comme son but était de soutenir l'effort de guerre, elle se devait d'être temporaire. Nous le savons aujourd'hui, les gouvernements ne l'ont jamais éliminée et elle est devenue, au fil du temps, un document très volumineux et très complexe à la suite des nombreux changements apportés. Nous en sommes aujourd'hui à un point où il devient pratiquement impossible pour la population en général d'en saisir toutes les subtilités. Il est généralement admis que les lois fiscales entrent dans la catégorie des lois les plus complexes à comprendre. Même pour un juriste, la lecture de ce texte de loi demeure très ardue.

Notre objectif premier sera de démontrer la difficulté de simplifier le système fiscal actuel. Pour ce faire, tout en démystifiant les concepts de crédit d'impôt et de déduction fiscale qui sont généralement très mal compris, nous illustrerons la complexité fiscale avec des exemples que les non- initiés peuvent aisément comprendre. Ensuite, nous saluerons quelques initiatives récentes de simplification, quoiqu'il y ait encore beaucoup de travail à faire à cet égard. Finalement, nous entamerons une réflexion sur des pistes de solution possibles.

\section{Précisions sur le fonctionnement du système fiscal}

\subsection{Le fonctionnement général d'un crédit d'impôt au fédéral}

Pour la majeure partie de la population, les subtilités d'un crédit d'impôt demeurent un grand mystère. Pour y voir plus clair, expliquons brièvement quelques éléments du calcul de l'impôt fédéral d'un particulier qui réside dans la province de Québec.

- La première étape consiste à prendre le revenu imposable du contribuable et d'y appliquer les différents taux d'imposition applicables. Par exemple, un particulier ayant un revenu d'emploi de $30000 \$$ sera assujetti à un taux de $15 \%$ puisque les premiers $45916 \$$ de revenu imposable sont imposés à ce taux en 2017. On obtient alors un montant d'impôt payable qui peut être réduit de différentes façons.

- Cette réduction se fait d'abord en appliquant une gamme de crédits d'impôt personnels.

\footnotetext{
a Professeur, MBA, M. Fisc., CPA, CA, Université du Québec à Chicoutimi

b Professeure, M.A.P., CPA, CA, Université du Québec à Chicoutimi 
L'économie d'impôt rattachée à un crédit correspond généralement au montant du crédit multiplié par un taux. Dans la plupart des cas, ce taux est de $15 \%$. À titre d'exemple, le crédit d'impôt pour l'achat d'une première habitation de $5000 \$$ amènera une réduction de l'impôt à payer de $750 \$(5000 \times 15 \%)$. Une fois tous ces crédits appliqués en réduction de l'impôt payable, on obtient alors un sous-total que l'on nomme l'impôt fédéral de base.

- On poursuit la réduction à l'aide de l'abattement d'impôt du Québec qui consiste à réduire l'impôt à payer de 16,5 \% de l'impôt fédéral de base. Il s'agit d'une diminution de l'impôt fédéral afin de tenir compte du fait que l'impôt provincial est perçu directement par le gouvernement provincial. En effet, contrairement aux résidents des autres provinces, ceux de la province du Québec ont une seconde déclaration de revenus à produire.
- Il y a d'autres éléments applicables, mais ceuxci ne seront pas pertinents pour illustrer le fonctionnement d'un crédit d'impôt.

Ce petit résumé sommaire devient nécessaire pour bien comprendre la valeur réelle de l'économie d'impôt rattachée à un crédit d'impôt fédéral pour un résident du Québec. Lorsque l'on combine le calcul du crédit d'impôt personnel avec l'abattement du Québec, on constate que l'économie correspond à $12,525 \%$ du montant du crédit ${ }^{1}$. Illustrons ces propos à l'aide de l'exemple du particulier ayant un revenu d'emploi de $30000 \$$ et pour lequel on applique le crédit d'impôt pour l'achat d'une première habitation de 5000 \$ainsi que l'abattement d'impôt du Québec. Nous omettons volontairement plusieurs subtilités afin de simplifier la démonstration de nos propos :

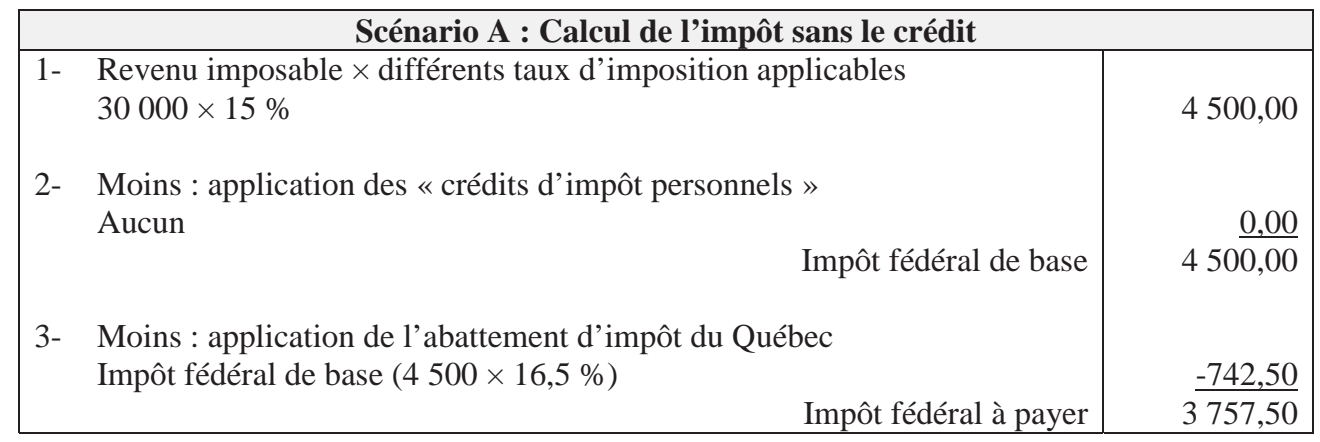

\begin{tabular}{|c|c|}
\hline \multicolumn{2}{|l|}{ Scénario B : Calcul de l'impôt avec le crédit } \\
\hline $\begin{array}{l}\text { 1- Revenu imposable } \times \text { différents taux d'imposition applicables } \\
30000 \times 15 \%\end{array}$ & 4500,00 \\
\hline $\begin{array}{l}\text { 2- Moins : application des «crédits d'impôt personnels » } \\
\text { Achat d'une première habitation }(5000 \times 15 \%)\end{array}$ & $-750,00$ \\
\hline Impôt fédéral de base & 3750,00 \\
\hline 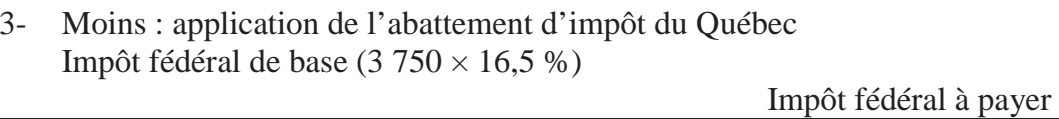 & $\frac{-618,75}{131,25}$ \\
\hline
\end{tabular}

L'écart entre les deux scénarios de 626,25 \$ (3 757,50 - 3 131,25) s'explique par l'utilisation d'un crédit d'impôt de $5000 \$$ qui rapporte une économie d'impôt de $626,25 \$(5000 \times 12,525 \%)$.

L'économie d'impôt rattachée à un crédit d'impôt personnel (626,25 \$ dans notre exemple) est donc un concept très différent du montant maximal du crédit (5 $000 \$$ dans notre exemple). Les politiciens exploitent habilement cette nuance afin de faire miroiter des économies d'impôt beaucoup plus grandes qu'elles ne le sont en réalité. Utilisons le cas du crédit d'impôt pour frais d'adoption pour le démontrer. Voici comment le gouvernement conservateur s'y est pris pour aviser la population de la 
bonification de ce crédit : «Le Plan d'action économique de 2014 a fait passer de $11774 \$$ à 15000 \$ le montant maximum des dépenses admissibles aux fins du crédit d'impôt pour frais d'adoption, rehaussant ainsi cet allègement fiscal pour mieux tenir compte des coûts particuliers liés à l'adoption d'un enfant $»^{2}$. Cette hausse de $3226 \$$ du montant maximal du crédit représente en réalité une économie d'impôt de $404 \$^{3}$ au fédéral. Toujours en lien avec ce crédit, lors de la campagne électorale fédérale de 2015, le gouvernement Harper proposait de bonifier davantage ce crédit et mentionnait ceci dans une publicité : «[...] but adoption costs can be high, that's why our party will increase the adoption fee expense tax credit of up to $\$ 20,000$ to make adoption more affordable for these carrying canadian family (sic) $»^{4}$.

L'accent est mis volontairement sur le montant maximal du crédit plutôt que sur la valeur réelle de l'économie d'impôt qu'il procure. Il faut bien comprendre qu'il n'y a aucune fausse information dans ces communications. Toutefois, la réalité est qu'une partie de la population croira que l'économie d'impôt correspond au montant du crédit. On peut comprendre les raisons d'une telle façon de procéder, mais cela contribue grandement à la confusion chez les contribuables.

Par ailleurs, bien que le taux accordé aux crédits soit généralement de $15 \%$ au fédéral et de $16 \%^{5}$ au provincial, il y a un grand nombre d'exceptions, et ce, principalement au provincial. À titre d'exemple, il s'élève à $10 \%$ pour les cotisations syndicales, à $50 \%$ pour les frais d'adoption alors qu'il varie selon le revenu familial pour les frais de garde.

D'autre part, le fait que les crédits d'impôt n'ont pas toutes les mêmes caractéristiques est aussi un facteur qui rend la fiscalité plus complexe. En effet, il y a des crédits d'impôt non remboursables, d'autres qui sont remboursables ou encore reportables. Dans le cas d'un crédit non remboursable, un contribuable qui n'a pas d'impôt à payer perdra définitivement les économies d'impôt liées à ces crédits. Les crédits pour l'achat d'une habitation ou pour les frais d'adoption sont des exemples de crédits d'impôt non remboursables au fédéral. Ainsi, le contribuable qui a acquis une première habitation, mais qui n'a pas d'impôt à payer, ne pourra obtenir le 750 \$ d'économie d'impôt auquel il aurait eu droit s'il avait eu un impôt payable, alors qu'il a bel et bien acquis une habitation admissible. Par contre, il existe quelques crédits non remboursables qui sont reportables tels que les crédits pour frais de scolarité ou pour dons. Dans ce cas, ce crédit n'est pas perdu et le contribuable pourra en bénéficier dans une année ultérieure, lorsqu'il aura suffisamment d'impôt à payer. En ce qui concerne les crédits d'impôt remboursables, comme c'est le cas pour le crédit pour les travaux de rénovation écoresponsable ou celui pour les frais de garde au provincial, il est possible de récupérer le montant du crédit admissible, qu'il y ait de l'impôt à payer ou non.

De plus, il faut aussi savoir que les modalités relatives aux crédits évoluent au fil du temps. Nous n'avons qu'à penser au crédit pour nouveau diplômé travaillant dans une région ressource éloignée qui, d'abord, était un crédit remboursable pour ensuite devenir non remboursable en plus d'avoir été rehaussé de 2000 \$ quelques années plus tard.

\subsection{Distinction entre un crédit d'impôt et une déduction du revenu imposable}

Il arrive souvent que les contribuables confondent les notions de crédit d'impôt et de déduction du revenu imposable. À l'instar du crédit d'impôt qui vient diminuer l'impôt payable, la déduction est un montant qui vient diminuer directement le revenu imposable. La cotisation à un régime enregistré d'épargne-retraite (REER) est un bon exemple de déduction autant au niveau fédéral que provincial. Ainsi, le contribuable ayant un revenu de $30000 \$$ qui demande une déduction pour un REER de $5000 \$$ aura donc un revenu imposable de $25000 \$$. L'économie qui résulte de cette déduction se calcule en multipliant le montant déductible avec le taux d'impôt marginal qui s'applique au revenu du contribuable. On constate que l'économie d'impôt d'une déduction varie en fonction du niveau de revenu du contribuable alors que celle du crédit sera constante, peu importe le revenu.

Pour les Québécois, la complexité fiscale se trouve amplifiée du fait qu'il arrive qu'un élément qui est admissible à une déduction au fédéral soit plutôt admissible à un crédit d'impôt au provincial. Les frais de garde ou encore les cotisations syndicales en sont de bons exemples. 


\section{Des exemples actuels ou passés de complexité fiscale}

\subsection{L'instauration du crédit d'impôt pour manuels}

L'instauration du crédit d'impôt pour manuels est la parfaite démonstration où le gouvernement a fait compliqué, alors qu'il aurait pu faire beaucoup plus simple. En 2006, le gouvernement conservateur de Stephen Harper proposait l'instauration d'un nouveau crédit d'impôt pour manuels afin d'encourager les étudiants à faire des études postsecondaires. Rappelons les différents crédits rattachés aux études alors en place :

1) Le crédit d'impôt pour frais de scolarité qui proposait une économie d'impôt en fonction des frais de scolarité payés relativement à des études postsecondaires (les frais d'admission, les frais d'examen, etc.).

2) Le crédit d'impôt pour études qui avait pour objectif de reconnaître que l'étudiant engage différents frais connexes (transport, stationnement, logement, etc.). La valeur du crédit dépendait du choix de l'étudiant de poursuivre un programme d'études postsecondaires à temps plein ou à temps partiel. Ce montant correspondait à $400 \$$ par mois si l'étudiant était à temps plein et à $120 \$$ par mois s'il était à temps partiel.

Dans son discours du budget, le ministre des Finances, monsieur Jim Flaherty, mentionnait :

Monsieur le Président, une bonne éducation est la clé d'un grand avenir pour nos jeunes Canadiens. Elle permet aussi au pays de compter sur une main-d'œuvre prête à travailler fort et à progresser. Pour encourager les Canadiens à faire des études postsecondaires [...] Nous allons établir un crédit d'impôt pour manuel (sic), qui profitera à environ 1,9 million d'étudiants canadiens, au coût de 260 millions de dollars sur deux ans ${ }^{6}$.

Ce nouveau crédit adoptait la même structure que le crédit d'impôt pour études déjà en place : $65 \$$ par mois si l'étudiant était à temps plein et 20 \$ par mois s'il était à temps partiel ${ }^{7,8}$.
La question légitime que l'on peut se poser est la suivante : si un crédit visant les frais connexes liés aux études existait déjà, pourquoi le gouvernement n'a-t-il pas seulement augmenté les seuils de 400 \$ à $465 \$$ pour un étudiant inscrit aux études à temps plein et de 120 \$à 140 \$ pour celui à temps partiel? On a complexifié le régime fiscal en ajoutant un nouveau crédit d'impôt parce qu'il était beaucoup plus payant politiquement d'instaurer une nouvelle mesure fiscale pour les étudiants que de bonifier une mesure déjà en place?

\subsection{La baisse du crédit d'impôt pour frais de scolarité}

À l'issue du Sommet sur l'enseignement supérieur qui s'est tenu en février 2013, le Parti Québécois dirigé par Pauline Marois a proposé d'abaisser le taux du crédit pour frais de scolarité de $20 \%$ à $8 \%$ à compter du $1^{\text {er }}$ mai $2013^{10}$. Rappelons brièvement les faits à l'origine de ce choix politique.

Tout débute lorsque le Parti libéral de Jean Charest propose une hausse des droits de scolarité de 325 \$ par année durant cinq ans à compter de l'automne 2012. Le but était alors que les étudiants paient leur juste part du plan de financement des universités ${ }^{11}$. Cette proposition a été l'élément déclencheur de la grève étudiante de 2012, mouvement social souvent nommé le " printemps érable ». Devant son incapacité à régler le conflit, le premier ministre Jean Charest annonce le déclenchement d'une élection le 4 septembre 2012 afin que la population tranche définitivement sur cette question. C'est ainsi que le Parti Québécois, dirigé par Pauline Marois, prend le pouvoir en promettant d'annuler la hausse des frais de scolarité proposée par les libéraux et en proposant la tenue d'un Sommet sur l'enseignement supérieur afin d'en arriver à un consensus. Parmi les différentes conclusions du Sommet, le Parti Québécois a finalement tranché pour une indexation des droits de scolarité selon le taux de croissance du revenu disponible des familles, soit environ $3 \%$. Il a aussi proposé la baisse du crédit d'impôt pour frais de scolarité ainsi que l'augmentation du seuil de contribution parentale à $45000 \$$ dans les paramètres du calcul de l'aide financière aux études afin de bonifier le régime de prêts et bourses. 
Certains argumenteront que cette baisse du crédit d'impôt s'apparente, avec quelques bémols, à une hausse des frais de scolarité de $18 \%$. Cette démonstration sera faite à l'aide des deux prochains tableaux en prenant l'hypothèse que les droits de scolarité annuels sont d'un montant de $2000 \$ 12$.

\begin{tabular}{|lr|}
\hline \multicolumn{2}{|c|}{ Scénario A : Hausse des droits de scolarité de 18 \% sans aucun changement } \\
sur le crédit d'impôt pour frais de scolarité
\end{tabular}

\begin{tabular}{|lr|}
\hline Scénario B : Aucune hausse des droits de scolarité et baisse du crédit d'impôt \\
pour frais de scolarité provincial de $20 \%$ à 8 \% \\
\hline Droits de scolarité annuels \\
Économie d'impôt liée au crédit fédéral (12,5\%) en tenant compte de & 2000 \\
l'abattement & -250 \\
Économie d'impôt liée au crédit provincial (8 \%) & -160 \\
Coût réel pour l'étudiant & 1590 \\
\hline
\end{tabular}

Puisque le coût réel pour l'étudiant demeure pratiquement identique, pouvons-nous réellement conclure que la baisse du crédit d'impôt s'apparente à une hausse des droits de scolarité de $18 \%$ ? En fait, ce n'est pas aussi simple que ça.

Le premier bémol à faire ressortir est le « moment » où la baisse du crédit d'impôt se fera ressentir. Ce crédit est un crédit non remboursable. Cela signifie que l'étudiant pourra utiliser le crédit uniquement si son impôt est suffisamment élevé. Or, les étudiants ont souvent des revenus faibles qui rendent impossible l'utilisation immédiate du crédit d'impôt. Dans ce cas, deux choix sont possibles. L'étudiant peut reporter à plus tard l'utilisation du crédit, ce qui correspond généralement au moment où il aura intégré officiellement le marché du travail et que ses revenus seront suffisamment élevés pour utiliser le crédit. L'autre option possible est de transférer le crédit à son conjoint, ses parents ou ses grands-parents. Ce scénario est généralement préconisé lorsque les droits de scolarités ne sont pas assumés par l'étudiant. On constate que la baisse du crédit n'est pas exactement la même chose qu'une hausse des droits de scolarité. La hausse aura un effet immédiat pour l'étudiant alors que l'effet de la baisse du crédit se fera généralement ressentir quelques années plus tard, dans le cas où il reporte son crédit. Si l'on admet que c'est durant les études que les besoins financiers sont les plus criants, la baisse du crédit est donc plus adaptée à cette réalité.

L'autre élément important à prendre en considération, c'est que la baisse du crédit d'impôt avait pour objectif de dégager des sommes supplémentaires afin de bonifier le régime de prêts et bourses. Au même moment où le gouvernement réduisait le crédit, il haussait graduellement le seuil de la contribution parentale qui entre dans le calcul de l'aide financière aux études pour le faire passer de $28000 \$$ à 45000 \$. Le but n'était donc pas de baisser le crédit pour «s'apparenter» à une hausse, mais bien pour financer la bonification de l'aide financière aux études.

Il était donc difficile pour les étudiants de bien comprendre toutes ces subtilités. Il fallait avoir le portrait global : d'un côté, il y a le crédit d'impôt et de l'autre, il y a l'aide financière aux études. Il était pourtant essentiel de les voir ensemble afin de bien comprendre le débat. Nous sommes d'avis que la baisse du crédit ne s'apparente pas à une hausse des droits de scolarité de $18 \%$, mais nous pouvons concevoir qu'il n'était pas si évident, pour la population en général, de bien cerner les différents enjeux. 


\subsection{La bonification de la prestation universelle pour la garde d'enfants}

La prestation universelle pour la garde d'enfants (PUGE), qui a été abolie en 2016, était un montant fixe que le gouvernement fédéral remettait mensuellement aux familles, ayant un ou plusieurs enfants de moins de 18 ans. En période préélectorale de juillet 2015, le gouvernement conservateur de Stephen Harper a bonifié de façon importante le montant mensuel de la PUGE. Il est passé de $100 \$$ à $160 \$$ pour les enfants de moins de 6 ans. De plus, un montant de $60 \$$ a été ajouté pour les enfants de 6 à 17 ans. En 2015, une famille recevait donc $720 \$$ de plus par enfant par rapport à 2014 .

Ceci n'est cependant pas tout à fait exact, car la PUGE était imposable. Il fallait donc payer de l'impôt sur les 720 \$ supplémentaires reçus. Pour un couple ayant un revenu familial de 70000 \$, le montant supplémentaire reçu était plutôt d'environ 515 \$ par enfant, une fois l'impôt payé13. Plus le revenu familial était élevé, plus ce montant diminuait. Pour un couple ayant un revenu familial de 90000 \$, le surplus reçu était plutôt d'environ $445 \$ 14$.

Au même moment, le gouvernement conservateur avait annoncé l'abolition du crédit pour enfant à charge auquel les familles avaient droit lors de la production de leur déclaration fiscale. Ce crédit leur permettait de retirer un montant de $282 \$ 15$ par enfant à charge de moins de 18 ans. Une fois l'impôt payé sur la PUGE et le crédit aboli, il restait finalement environ $233 \$$ de plus par enfant pour la famille qui gagnait $70000 \$$ annuellement et $163 \$$ pour celle qui avait un revenu annuel de $90000 \$$.

La complexité du système ne permettait pas aux familles de bien comprendre l'impact monétaire réel dans leur poche. Ainsi, elles pouvaient concrètement voir les effets positifs de la bonification en recevant mensuellement un montant supplémentaire de $60 \$$ directement dans leur compte bancaire. Ce qui était plus difficile à percevoir était les effets plus négatifs liés à ces mesures, soit l'impôt à payer sur ce montant et la disparition du crédit pour enfant à charge, qui ne se faisaient sentir que lors de la production de la déclaration de revenus. Les familles avaient généralement eu le temps de dépenser les 720 \$ reçus avant même de devoir payer la facture fiscale liée à cette somme.

\subsection{Les frais de garde et la contribution additionnelle}

C'est en 1997, avec l'annonce de la Politique familiale par la ministre Pauline Marois, qu'est introduit le concept des " garderies à $5 \$$ par jour». L'instauration d'un tel réseau visait l'égalité des chances pour les enfants et l'accessibilité au marché du travail pour les femmes afin de faire reculer la pauvreté. Depuis leur apparition, les places à 5 \$ ont été augmentées à $7 \$$ en 2003 , avec l'arrivée au pouvoir du Parti libéral de Jean Charest, pour ensuite être indexées à 7,30 \$ en octobre 2014, sous le gouvernement libéral de Philippe Couillard. Le montant continuera d'être indexé annuellement par la suite.

Au départ, l'instauration des garderies à contribution réduite a eu son lot de contestation :

Le gouvernement semble vouloir nationaliser le système de garderie alors que les garderies privées et le milieu informel de garde (assuré, par exemple, par la famille ou les voisins) s'avèrent nettement moins coûteux et plus souples. [...] Il aurait été plus efficace de simplement transférer les sommes considérables en jeu à toutes les familles et de leur laisser le choix du mode de garde de leurs enfants. Contrairement au programme d'allocation à la naissance, le programme des garderies à $5 \$$ n'est pas un programme universel : il pénalise les familles qui n'utilisent pas le système ${ }^{16}$.

Par la suite, il a tout de même été démontré que cette mesure a eu des retombées économiques importantes. En effet, tel que le relate le journaliste Gérald Fillion :

Son apport économique depuis plus de 15 ans semble faire consensus autour de la table de la commission de révision des programmes. Robert Gagné, Claude Montmarquette et les autres commissaires affirment que les garderies à $5 \$$ et $7 \$$ ont permis à des dizaines de milliers de femmes d'intégrer le marché du travail. Selon la commission Robillard, le pro- 
gramme des garderies à tarif réduit « a contribué directement à accroittre la présence des femmes sur le marché du travail »17.

Évidemment, plus il y a de femmes sur le marché du travail, plus il y a d'impôts et de taxes à la consommation perçues par le gouvernement. Cela autofinance une portion des coûts engendrés par le programme.

En avril 2015, de grands changements se produisent avec l'apparition de la contribution additionnelle. Mais d'abord, illustrons le coût journalier pour les familles qui utilisaient un service de garde avant cette réforme. Il est important ici de préciser que lorsque l'enfant fréquente un service de garde dit subventionné, le montant déboursé, bien qu'admissible à la déduction pour frais de garde au fédéral, n'est pas admissible au crédit pour frais de garde au provincial. Évidemment, les frais déboursés pour la garde d'un enfant dans un service non subventionné demeurent admissibles au crédit provincial. Il faut savoir aussi que le taux du crédit accordé est variable en fonction du revenu familial. En effet, plus le revenu est faible, plus le taux accordé est généreux. Ainsi, comparons le coût journalier des frais de garderie payés en $2015^{18}$, pour un couple ayant un revenu familial de 90000 \$, selon quel'enfant fréquentait une garderie subventionnée ou non.

\begin{tabular}{|ll|r|}
\hline \multicolumn{2}{|c|}{$\begin{array}{c}\text { Scénario A : Calcul du coût journalier lié aux frais de garde } \\
\text { Garderie subventionnée - 7,30 \$ par jour }\end{array}$} \\
\hline $1-$ & Frais de garde payé & 7,30 \\
$2-$ & $\begin{array}{l}\text { Moins : déduction pour frais de garde pour enfants au fédéral } \\
7,30 \text { \$ } 12,525 \% \text { (revenu individuel de } 45000 \text { \$, taux d'imposition de } 15 \%)\end{array}$ \\
& $\frac{-0,91}{6,39}$ \\
3- & $\begin{array}{l}\text { Moins : crédit pour frais de garde au provincial } \\
\text { Non admissible }\end{array}$ & $\underline{-0,00}$ \\
\hline
\end{tabular}

\begin{tabular}{|c|c|}
\hline \multicolumn{2}{|l|}{$\begin{array}{l}\text { Scénario B : Calcul du coût lié aux frais de garde } \\
\text { Garderie non subventionnée - } 30 \text { \$ par jour }\end{array}$} \\
\hline 1- Frais de garde payé (hypothèse) & 30,00 \\
\hline 2- Moins : déduction pour frais de garde pour enfants au fédéral & \\
\hline $30 \$ \times 12,525 \%$ (revenu individuel de $45000 \$$, taux d'imposition de $15 \%$ ) & $\frac{-3,76}{2,21}$ \\
\hline 3- Moins : crédit pour frais de garde au provincial & \\
\hline $30 \$ \times 60 \%$ & $\frac{-18,00}{8,24}$ \\
\hline
\end{tabular}

À partir de l'exemple précédent, il est possible de constater qu'à un certain niveau de revenu familial (ici, un revenu de 90000 \$ réparti également entre les conjoints), il n'en coûtait pas beaucoup plus cher d'envoyer son enfant dans une garderie non subventionnée coûtant $30 \$$ par jour que dans celle à 7,30 \$ par jour. Il y a fort à parier que les familles n'étaient pas toutes conscientes de cela. Il demeure difficile pour ceux qui ne connaissent pas les particularités de notre système fiscal de calculer le coût réel des frais de garde selon leur situation. La plupart auront tendance à comparer seulement le $30 \$$ avec le 7,30 \$. Par contre, il est important de spécifier que plus le revenu familial augmente, plus l'écart se creuse, car le crédit accordé au provincial sera moins élevé. D’ailleurs, ayant conscience de cette complexité, le gouvernement a élaboré un outil disponible en ligne ${ }^{19}$ afin d'aider les familles à déterminer le coût quotidien d'une place en garderie selon leur situation.

L'instauration de la contribution additionnelle en avril 2015, par le ministre Leitao, est loin de simplifier la chose et les familles étaient très mal préparées à payer la facture qui les attendait lors de la production de leur déclaration fiscale au printemps 2016. La mesure de cette contribution, 
dite modulée, se base sur le revenu familial de l'année précédente et exige le paiement d'une contribution additionnelle journalière aux parents qui utilisent un service de garde subventionné. Le calcul et le paiement s'effectuent directement par le biais de la déclaration fiscale. Prenons l'exemple de la famille avec un enfant qui avait un revenu familial de 90000 \$. Elle s'est vu réclamer un montant supplémentaire de $2,95 \$ 20$ par jour pour la contribution additionnelle ce qui portait la facture à plus de $465 \$$ pour $2015^{21}$ et payable au 30 avril 2016. Considérant l'économie liée à la déduction admissible au fédéral, il en résultait donc un coût journalier de $8,97 \$(7,30+2,95-(10,25 * 12,525 \%))$, comparativement à 6,39 \$ avant la réforme.

Depuis cette réforme, il a même été démontré que dans certaines situations familiales, il s'avère plus avantageux financièrement d'utiliser un service de garde non subventionné. Bien que cela implique de devoir payer un tarif quotidien beaucoup plus élevé, il est alors possible de profiter du crédit d'impôt au Québec et d'une déduction plus importante au fédéral. Cela demeure accessible puisqu'il existe un programme de versements anticipés pour ce crédit, ce qui facilite la gestion des liquidités pour les familles qui choisissent cette option.

En conclusion, l'ensemble des exemples précédents démontrent clairement à quel point la compréhension de notre système fiscal peut s'avérer une tâche ardue. Peu importe les raisons qui ont mené à cette complexification, qu'elles soient de nature politique ou autres, il en demeure un constat indéniable, il est difficile d'y voir clair lorsque l'on n'est pas formé dans le domaine. On peut toutefois s'encourager de certaines initiatives récentes prises par les deux paliers de gouvernement.

\section{Les initiatives récentes menant vers une simplification}

\subsection{L'instauration de l'Allocation canadienne pour enfants}

Les libéraux de Justin Trudeau concrétisent une promesse électorale dans le budget 2016 en instaurant l'Allocation canadienne pour enfants (ACE) à partir de juillet 2016. Cette allocation prétend être plus simple, libre d'impôt, mieux ciblée et beaucoup plus généreuse ${ }^{22}$.

On peut dire que l'objectif de simplicité est atteint puisque l'ACE remplace plusieurs anciennes mesures destinées aux familles : la Prestation universelle pour la garde d'enfants, la Prestation fiscale canadienne pour enfants, le Supplément de la prestation nationale, la baisse d'impôt pour les familles (fractionnement du revenu pour les familles avec enfants mineurs), le crédit d'impôt pour la condition physique des enfants et le crédit d'impôt pour les activités artistiques des enfants. Elle représente une amélioration certaine pour plusieurs familles canadiennes. Seules les familles plus fortunées recevront des prestations réduites par rapport à l'ancien régime ${ }^{23}$.

Du point de vue de la complexité fiscale, le remplacement de l'ancien régime par celui de l'ACE est une excellente nouvelle. Plutôt que d'avoir plusieurs mesures fiscales destinées aux familles et aux enfants, on les consolide afin de n'en présenter qu'une seule qui se veut simple, libre d'impôt et mieux ciblée. Toutefois, il reste encore du travail à faire puisqu'il s'avère qu'elle n'est pas réellement plus généreuse à long terme.

En effet, les chercheurs Godbout et St-Cerny de la Chaire en fiscalité et en finances publiques de l'Université de Sherbrooke ont noté l'absence d'indexation des paramètres de l'ACE. L'indexation à l'inflation était pourtant prévue lors de la promesse électorale ${ }^{24}$. Parmi les mesures qu'elle remplace, la Prestation fiscale canadienne pour enfants et le Supplément de la prestation nationale étaient, quant à elles, indexées. Le gouvernement Trudeau a donc remplacé certaines mesures indexées par une seule mesure non indexée. Sans un mécanisme d'indexation, l'avantage de l'ACE par rapport aux anciennes mesures s'affaiblira d'année en année. En effet, le chercheur Godbout explique très bien l'impact de cette absence dans une entrevue accordée au journal Le Devoir : "L'inflation aura comme effet que votre chèque va perdre de la valeur avec le temps. Si le coût de la vie augmente, si votre salaire augmente avec le coût de la vie, le montant de la prestation devient de moins en moins efficace pour combattre la pauvreté $»^{25}$. 
À ce stade, il est très légitime de se demander pourquoi une telle inflation n'a pas été prévue. Les libéraux ne peuvent même pas prétendre à un oubli, car l'indexation était initialement prévue dans la plateforme électorale. Ils ont donc volontairement enlevé cette indexation. Est-ce qu'un contribuable qui n'a aucune connaissance particulière en fiscalité aurait pu raisonnablement détecter une telle subtilité? La réponse est évidemment négative. Heureusement que les chercheurs de l'Université de Sherbrooke ont soulevé le problème, obligeant ainsi le gouvernement Trudeau à répondre aux questions des journalistes. Ils ont alors annoncé leur intention d'indexer l'ACE à compter de 202026. Il est dommage qu'une belle initiative de simplification fiscale ait été entachée par l'absence d'un mécanisme d'indexation.

\subsection{La consolidation des crédits pour les aidants naturels dans la législation fédérale}

En 2016, il existait trois crédits d'impôt non remboursables visant les aidants naturels. Il y avait le crédit pour personnes à charge ayant une déficience, le crédit pour aidants naturels et le crédit d'impôt pour aidants familiaux. Un aidant naturel qui avait à sa charge une personne admissible pouvait la désigner dans sa déclaration de revenus et obtenir une économie d'impôt. Il y avait toutefois des règles complexes. En effet, une personne à charge pouvait se qualifier à plusieurs de ces crédits, mais il n'était pas possible de tous les cumuler. Il y avait donc une optimisation à effectuer. Celle-ci devait tenir compte de la valeur maximale de chacun des crédits (qui était variable) ainsi que du revenu de la personne à charge admissible qui pouvait réduire cette valeur. Les seuils de revenu amenant cette réduction étaient eux aussi variables d'un crédit à l'autre.

Le budget fédéral de 2017 propose d'éliminer ces trois crédits afin de les remplacer par un nouveau crédit canadien pour aidant naturel. L'effet net sera de simplifier et d'améliorer les mesures fiscales pour les aidants naturels 27 .

\subsection{Le calcul des crédits d'impôt personnels de la législation québécoise}

Outre le Québec, le fédéral et les autres provinces calculent l'économie d'impôt rattachée à un crédit d'impôt personnel en utilisant le taux applicable à la première tranche de revenu imposable de la table d'impôt. Notre province était la seule à utiliser celui de la deuxième tranche de revenu imposable. Étant donné que les taux des première et deuxième tranches de revenu imposable sont respectivement de $16 \%{ }^{28}$ et de $20 \%$, il était compliqué pour les contribuables d'établir l'équivalence du crédit en termes de revenu imposable.

À titre d'exemple, le crédit « montant personnel de base » était de 11550 \$ en 2016. Il procurait donc une économie d'impôt de 2310 \$ $(11550 \times 20 \%)$. Comme le crédit utilise le taux de la deuxième tranche de revenu imposable, il fallait appliquer un facteur de $1,25^{29}$ afin d'établir le niveau de revenu imposable qu'il permettait d'éliminer. Autrement dit, le crédit « montant personnel de base » permettait d'éliminer un revenu imposable de $14438 \$(11550 \times 1,25)$ en 2016. En effet, un contribuable qui affiche un revenu imposable de ce montant payait 2310 \$ d'impôt $(14438 \times 16 \%)$ selon la table, puisque les premiers 42390 \$ de revenu imposable étaient imposés à $16 \%$ en 2016.

\begin{tabular}{|l|r|}
\hline \multicolumn{1}{|c|}{ Illustration des propos } \\
\hline $1-\quad$\begin{tabular}{l}
\multicolumn{1}{|c|}{ Revenu imposable $\times$ différents taux d'imposition applicables } \\
$14438 \times 16 \%$
\end{tabular} & 2310 \\
2- & $\begin{array}{l}\text { Moins : application du crédit «montant personnel de base » } \\
11550 \times 20 \%\end{array}$ \\
\end{tabular}

Il était donc difficile pour le contribuable moyen de comprendre qu'un crédit d'un montant de 11550 \$ permettait d'éliminer un revenu imposable de 14438 \$. Voici ce que le gouvernement a proposé afin de simplifier le tout :
Aussi, pour faciliter la compréhension par la population des règles de base du régime d'imposition québécois, des modifications seront apportées à la législation fiscale pour faire en sorte que le taux utilisé aux fins du calcul des 
crédits d’impôt personnels corresponde dorénavant au taux applicable à la première tranche de revenu imposable de la table d'impôt des particuliers, soit $16 \%$, sans pour autant que la valeur de chacun de ces crédits soit réduite ${ }^{30}$.
Afin d'atteindre cet objectif, le montant des différents crédits d'impôt personnels a été augmenté afin que l'économie d'impôt demeure la même. À titre d'exemple, voici de quelle façon cela se traduit sur le crédit "montant accordé en raison de l'âge $»^{31}$ :

\begin{tabular}{lcccccc} 
& \multicolumn{2}{c}{$\begin{array}{c}\text { Taux de conversion de } \\
\mathbf{2 0} \% \text { avant budget }\end{array}$} & & \multicolumn{2}{c}{$\begin{array}{c}\text { Taux de conversion } \\
\text { de } \begin{array}{c}\mathbf{1 6} \% \text { après } \\
\text { budget }\end{array}\end{array}$} \\
\cline { 2 - 3 } \cline { 6 - 7 } & $\begin{array}{c}\text { Montant } \\
\text { accordén }\end{array}$ & $\begin{array}{c}\text { Réduction } \\
\text { d'impôt }\end{array}$ & & $\begin{array}{c}\text { Montant } \\
\text { accordé }\end{array}$ & $\begin{array}{c}\text { Réduction } \\
\text { d'impôt }\end{array}$ \\
\hline Montant accordé en raison de l'âge & 2505 & 501 & & 3132 & 501 \\
\hline
\end{tabular}

Nous ne pouvons que saluer cet effort récent qui permet d'améliorer la compréhension par lapopulation des règles de base du système fiscal québécois. Toutefois, le souhait de ne pas affecter négativement l'économie d'impôt a un effet pervers. En effet, certains crédits d'impôt personnels se calculent à partir d'une somme déboursée. À titre d'exemple, le crédit pour frais médicaux se base sur le montant payé pour des médicaments sous prescription et non remboursé par une assurance. Le fait de faire passer le taux de ce crédit de $20 \%$ à $16 \%$ aurait nécessairement eu pour effet de réduire la valeur de l'économie d'impôt. Ainsi, le taux de crédit demeure à $20 \%$ pour les crédits personnels suivants : frais médicaux, frais pour soins médicaux non dispensés dans la région de résidence du particulier, intérêts payés sur un prêt étudiant et la première tranche de 200 \$ du crédit pour dons.

Il est un peu ironique de dire qu'en voulant simplifier les choses, on a introduit une série d'exceptions qui contribuent aussi à la complexification du système fiscal québécois.

\section{Réflexion sur le système fiscal actuel}

Tous s'entendent pour dire que le système fiscal actuel est complexe et qu'il l'est doublement pour le contribuable québécois. Autant il n'est pas facile à maittriser pour les professionnels en la matière, autant il devient préjudiciable pour les contribuables qui n'ont pas accès à toutes les ressources pour optimiser leur situation notamment lorsque le moment est venu de préparer leur déclaration de revenus. Cette complexité représente d'ailleurs un coût élevé autant pour le contribuable que pour l'administration publique. Par son inaccessibilité, il en résulte un effet d'opacité et le contribuable y voit un manque de transparence qui vient miner sa confiance envers le système.

Il est clair que la complexité du système n'est pas apparue du jour au lendemain et elle n'a certainement pas été consciemment souhaitée. Elle s'est développée graduellement d'une façon insidieuse. Sans avoir la prétention d'en expliquer toutes les causes, il semble que la recherche d'un bénéfice politique ait contribué à cela. Autant par les partis au pouvoir qui, en période préélectorale, ajoutent des mesures pour plaire, autant par ceux qui mettent en œuvre leurs promesses lorsqu'ils arrivent au pouvoir.

Il y a tout de même de nobles intentions de la part des gouvernements dont la fiscalité demeure un outil pour l'atteinte d'une certaine justice sociale. Cela les a amenés, au fil des années, à instaurer des mesures qui permettent de diminuer le fardeau fiscal de certains ou encore, de stimuler l'économie. Mais cela contribue naturellement à complexifier le système. Sans compter qu'en voulant simplement limiter les évitements et les abus ou réagir a posteriori à ceux-ci, les textes de loi sont régulièrement révisés et se voient donc complexifiés.

La simplification n'est évidemment pas si simple que cela. Bien que les gouvernements en soient conscients et semblent vouloir en faire une priorité, il reste que la lourdeur des structures en place ainsi que la réalité économique ne facilitent pas son épuration. À cet effet, nous pouvions lire dans un document produit par le ministère des Finances du Québec : 
Le principe de simplicité d'un régime fiscal repose sur la prémisse qu'un système simple à comprendre et à administrer est plus facile d'observation et moins coûteux, à tous égards. Or, bien que l'objectif visé par ce principe soit tout aussi important, son atteinte est souvent entravée au bénéfice des principes d'équité et de neutralité du régime, ainsi que pour d'autres considérations tout aussi légitimes ${ }^{32}$.

Cependant, le fait que ce soit difficile ne doit pas être une raison pour ne pas aller de l'avant. Il semble que l'on devrait en faire une priorité. D'ailleurs, dans son document, le ministère des Finances du Québec établit que lorsqu'un régime fiscal est simple et compréhensible, il est plus facile de s'y conformer et de l'administrer. En effet, il fait ressortir des avantages évidents de la simplification que nous pouvons résumer ainsi :

- Une réduction des coûts pour les contribuables, coûts engendrés par les ressources nécessaires pour comprendre l'application de la législation à l'égard de leur situation et pour se conformer à leurs obligations.

- Une réduction des coûts pour l'administration publique, coûts liés à la rédaction de la législation en perpétuelle évolution, pour l'interpréter et l'appliquer correctement, et pour expliquer les règles aux contribuables.

- Une diminution des besoins en vérification et des litiges qui en résultent.

- Une amélioration de la transparence provenant d'une meilleure compréhension du corpus législatif de la part des contribuables, favorisant ainsi une plus grande accessibilité et donc une plus grande confiance collective en son équité et son efficacité, espérant du même coup remarquer une diminution de l'économie souterraine.

Une piste de solution pourrait être la mise en place d'une quelconque forme de reddition de comptes. Une instance indépendante pourrait alors évaluer si la mesure fiscale répond réellement à l'objectif qui a mené à son application. À titre d'exemple, le gouvernement du Québec a instauré en 2003 le crédit d'impôt pour les nouveaux diplômés travaillant dans une région ressource éloignée afin de lutter contre l'exode des jeunes diplômés vers les grands centres urbains et les inciter à se rendre en région pour acquérir une première expérience de travail dans leur domaine de spécialisation :
Actuellement, peu de jeunes quittant leur région natale pour poursuivre des études spécialisées y reviennent pour y entreprendre leur carrière professionnelle. Cette migration des jeunes vers les grands centres urbains est un phénomène social des plus préoccupants pour l'avenir des régions, puisqu'il les affecte tant sur le plan démographique que sur les plans social et économique ${ }^{33}$.

On pourrait statuer sur une fréquence où il est obligatoire d'établir la pertinence d'une mesure fiscale. Ce qui était pertinent il y a dix ans ne l'est peut-être plus aujourd'hui. Le gouvernement aurait l'obligation de publier des statistiques sur l'utilité et l'efficacité d'un crédit ou d'une prestation. Il se peut que l'objectif soit noble, mais que le moyen utilisé pour l'atteindre soit inefficace. Selon les projections du ministère des Finances du Québec, le crédit pour nouveaux diplômés coûtera 48 millions de dollars à l'État québécois en $2016^{34}$. À titre d'exemple, la région administrative du Saguenay-Lac-Saint-Jean constitue une région ressource éloignée aux fins de ce crédit. On saurait alors dans quelle mesure le crédit a réellement incité les jeunes professionnels à s'établir dans cette région. À grande échelle, cet exercice permettrait probablement d'effectuer un écrémage et conduirait vers une simplification du système fiscal.

Il est entendu que la pertinence d'une mesure devrait s'évaluer autant sur une base quantitative que qualitative. Bien que nous référions à l'évaluation de l'atteinte de l'objectif, il demeure important de prendre en compte les coûts que l'instauration d'une mesure a pu susciter. Par exemple, bien qu'il ait été démontré que l'instauration des services de garde subventionnés a eu des retombées positives en ce qui concerne l'accessibilité des femmes sur le marché du travail et qu'il en découle une forme d'autofinancement, il demeure aussi pertinent de tenir compte des coûts de structure gouvernementale que cela a engendrés pour obtenir un portrait global des retombées.

Une autre piste de solution pourrait être de favoriser les mesures fiscales les plus simples. En effet, pour atteindre un objectif, différents outils sont à la portée du gouvernement : crédit d'impôt, déduction fiscale, prestation, exonérations fiscales, report d'impôt, etc. Certains de ces outils, comme la prestation, sont beaucoup plus simples à comprendre pour le contribuable. 
L'Allocation canadienne pour enfants est un exemple récent dont on devrait s'inspirer. On a favorisé une prestation non imposable au détriment de diverses mesures fiscales qui incluaient des crédits d'impôt plus complexes à comprendre.

\section{Conclusion}

Il serait utopique de croire que nous en arriverons un jour à un système fiscal vraiment simple à comprendre par l'ensemble des contribuables. Même si les nombreux avantages de la simplicité ont été avancés, d'autres grands principes, comme l'équité, la neutralité et la compétitivité fiscale sur le plan international, doivent également être considérés. Il en résulte qu'une certaine forme de complexité sera toujours présente et elle s'avère même nécessaire.
Toutefois, cela ne signifie pas qu'il ne faut pas faire d'efforts. Les quelques initiatives récentes présentées sont très encourageantes. La mise en place d'un système de reddition de comptes et l'adoption de mesures fiscales plus faciles à comprendre, comme l'Allocation canadienne pour enfants, font partie des recommandations afin de poursuivre cet objectif de simplicité. Puisque l'histoire nous apprend que la politique fiscale est fortement influencée par des considérations politiques plutôt que par des fondements théoriques, les gouvernements devront faire preuve d'une grande volonté si l'on veut collectivement réussir à « décomplexifier » la fiscalité.

\section{NOTES}

$1 \quad 15 \%-(16,5 \% \times 15 \%)=12,525 \%$

2 Ministère des Finances du Canada (2014). Nouvelles - Le gouvernement Harper aide à rendre l'adoption plus abordable.

$3(15000-11774) \times 12,525 \%$.

4 Increasing the adoption expense tax credit, en ligne : YouTube (chaîne officielle du parti conservateur). Repéré à https://www.youtube.com/watch?v=sopbkVhtIhA

5 Jusqu'à tout récemment, le taux était de $20 \%$ et suite à l'écriture de ces lignes, le taux a été modifié à $15 \%$ de façon rétroactive pour toute l'année d'imposition 2017 : Ministère des Finances du Québec (2017). Bulletin d'information 2017-11, Mesures annoncées à l'occasion de la présentation de la mise à jour du plan économique du Québec, p. 3.

6 Ministère des Finances du Canada (2006). Le budget de 2006 - Le discours du budget, p. 6-7.

7 Ministère des Finances du Canada (2006). Le budget de 2006 - Le plan budgétaire, p. 245.

8 Il est à noter que les deux crédits (pour études et pour manuels) n’existent plus depuis le 1er janvier 2017. Ministère des Finances du Canada (2006). Le budget de 2016 - Mesures fiscales : Renseignements supplémentaires, p. 17.

9 Sherman, D. (2010). Paragraphe 118.6(2.1) L.I.R. - Notes de David Sherman, 22e éd., Toronto, Ontario : Carswell.

10 Ministère des Finances du Québec (2013). Bulletin d'information 2013-3, Réduction du taux de transformation en crédit d'impôt des frais de scolarité et d'examen, p. 2.

11 Ministère des Finances du Québec (2011). Budget du Québec 2011-2012, Fascicule : Un plan de financement des universités équitables et équilibrées, p. 20.

12 Inspiré de l'article de Stéphanie Grammond «Les étudiants rattrapés par le fisc » paru le 19 mars 2014 dans La Presse.

13 En supposant que chaque membre du couple gagne annuellement 35000 \$ et donc que leur taux marginal d'impôt était de $28,53 \%$ (taux de 2015).

14 En supposant que chaque membre du couple gagne annuellement 45000 \$ et donc que leur taux marginal d'impôt était de $38,37 \%$ (taux de 2015).

15 En $2014: 2255 \$ \times 12,525 \%$.

16 Andries, P. (2003, 5 février). Les garderies à 5 \$ - Une politique familiale réservée aux « familles modèles »?, Le Devoir.

17 Fillion, G. (2014, 24 novembre). L'effet des garderies et les femmes [Blogue Économie de Radio-Canada.ca]. Repéré à http://blogues.radio-canada.ca/geraldfillion/2014/11/24/leffet-des-garderies-et-les-femmes/ 
18 Avant le 22 avril 2015, car le tarif a fait l'objet d'une réforme à compter de cette date.

19 Ministère des Finances du Québec, Cô̂t d'une place en service de garde. Repéré à http://www.budget.finances.gouv.qc.ca/Budget/outils/garde-net-fr.asp

$20(((90000 \$-75000 \$) \times 3,9 \%) / 260)+0,70 \$=2,95 \$$

21 Avec une hypothèse de 181 jours de garde à compter du 22 avril, multiplié par 2,95 \$, le montant s'élève à 534 \$. En considérant une économie liée à la déduction au fédéral, le montant s’élève à 467 \$ (534- (534 x 12,525\%)). Pour une année complète, cela aurait représenté environ 670 \$, en considérant 260 jours de garde.

22 Ministère des Finances du Canada (2016). Le budget de 2016 - Le plan budgétaire, p. 62.

23 Godbout, L. et St-Cerny, S. (2016). L'allocation canadienne pour enfants : de la promesse à sa mise en place (Cahier de recherche no 2016-09). Université de Sherbrooke, Chaire de recherche en fiscalité et en finances publiques, p. 24.

24 Godbout, L. et St-Cerny, S. (2016). L'allocation canadienne pour enfants : de la promesse à sa mise en place (Cahier de recherche no 2016-09). Université de Sherbrooke, Chaire de recherche en fiscalité et en finances publiques, p. 3-4.

25 Proulx, B. (2016, 29 juin). Une allocation canadienne pour enfants moins généreuse que promis. Le Devoir.

26 Ministère des Finances du Canada (2017). Le budget de 2017 - Le plan budgétaire, p. 301.

27 Ministère des Finances du Canada (2017). Le budget de 2017 - Mesures fiscales : Renseignements supplémentaires, p. 11.

28 Suite à l'écriture de ces lignes, le taux a été modifié à $15 \%$ de façon rétroactive pour toute l'année d'imposition 2017 : Ministère des Finances du Québec (2017). Bulletin d'information 2017-11, Mesures annoncées à l'occasion de la présentation de la mise à jour du plan économique du Québec, p. 3.

$291+((20 \%-16 \%) / 16 \%)=1,25$

30 Ministère des Finances du Québec (2017). Le budget 2017-2018 - Le plan économique du Québec (Renseignements additionnels 2017-2018), p. A.5.

31 Id., p. A.6.

32 Ministère des Finances du Québec (2004). Simplification de la fiscalité, p. 3.

33 Ministère des Finances du Québec (2003). Le budget 2003-2004 «non voté » - Renseignements additionnels sur les mesures du budget 2003-2004, p. 7

34 Ministère des Finances du Québec (2017). Dépenses fiscales - édition 2016, p. A.36. 